山્યFANÇAISE

$\supset \mathrm{DE}$

$\stackrel{1}{\simeq}$ PÉDAGOGIE

\section{Revue française de pédagogie}

Recherches en éducation

168 | juillet-septembre 2009

Enseignement et apprentissages, entre psychologie et didactiques

\title{
Soutien en lecture en troisième année de cycle 2 : évaluation de deux dispositifs contrastés
}

Extra help in reading in second grade: assessing two very different teaching devices

Apoyo a la lectura en tercer año de ciclo 2: evaluación de dos dispositivos contrastados

Nachhilfe beim Lesen in der 2. Klasse: Bewertung zweier unterschiedlicher

Vorrichtungen

\section{Saint-Cyr Chardon}

\section{(2) OpenEdition}

\section{Journals}

Édition électronique

URL : http://journals.openedition.org/rfp/1723

DOI : $10.4000 /$ rfp. 1723

ISSN : 2105-2913

Éditeur

ENS Éditions

Édition imprimée

Date de publication : 1 juillet 2009

Pagination : 19-37

ISBN : 978-2-7342-1172-3

ISSN : 0556-7807

Référence électronique

Saint-Cyr Chardon, « Soutien en lecture en troisième année de cycle 2 : évaluation de deux dispositifs contrastés », Revue française de pédagogie [En ligne], 168 | juillet-septembre 2009, mis en ligne le 01 juillet 2013, consulté le 09 septembre 2019. URL : http://journals.openedition.org/rfp/1723 ; DOI : 10.4000/rfp. 1723 


\section{Soutien en lecture en troisième année de cycle 2 : évaluation de deux dispositifs contrastés \\ Saint-Cyr Chardon}

Cette recherche, conduite en contexte scolaire auprès de trois groupes de faibles décodeurs en lecture de troisième année de cycle 2 (CE1), évalue les effets sur le décodage et la compréhension d'un programme d'entraînement en lecture composé d'exercices visant à parfaire les mécanismes de reconnaissance des mots écrits, la lecture orale et la maîtrise des correspondances entre graphèmes et phonèmes. Constitué de onze séances dispensées à raison d'une heure par semaine, ce programme est mis en œuvre en faisant appel à deux dispositifs pédagogiques distincts : soit les élèves travaillent seuls, soit ils travaillent par équipe de deux. Les performances en lecture orale, dictée et compréhension en lecture silencieuse sont mesurées avant et après l'entraînement. Au post-test, des effets positifs sont relevés en lecture à voix haute et en dictée mais également, et c'est le résultat le plus important, en compréhension. De plus, un post-test différé effectué quatre mois après la fin de l'entraînement indique que les progrès se maintiennent dans le temps. Par contre, hormis pour l'épreuve de dictée, l'entraînement ne semble pas suffisant pour provoquer une réduction significative des écarts constatés au prétest entre faibles décodeurs et décodeurs sans difficulté. Concernant l'effet d'un dispositif particulier de mise en œuvre, aucune différence significative n'est relevée entre les performances des élèves qui ont travaillé seuls ou à deux. Ce dernier résultat pourrait s'expliquer, outre le jeune âge des élèves, par le contenu didactique des séances, peu susceptible de provoquer échanges et coordinations de points de vue car trop éloigné de réelles situations problèmes qui seraient plus particulièrement propices au travail de groupe.

Descripteurs (TESE) : école primaire, difficultés de lecture, pédagogie de soutien, méthode d'enseignement.

\section{INTRODUCTION}

Il est convenu de dire qu'au terme de la scolarité primaire, tous les élèves devraient avoir acquis une maîtrise suffisante de la lecture pour aborder le collège dans des conditions satisfaisantes, l'objectif principal n'étant plus à ce niveau d'« apprendre à lire » mais de "lire pour apprendre » (Leach, Scarborough \& Rescorla, 2003, p. 211). Or l'observation des évaluations nationales montre qu'à l'entrée en $6^{\mathrm{e}}$ un tiers seulement des élèves dominent les compétences attendues. Dans le même temps, un autre tiers de ces 
collégiens éprouvent encore de notables difficultés et parmi eux, $14 \%$ s'avèrent rapidement démunis lorsque le document à lire va au-delà d'un bref paragraphe (Rémond, 2007 ; Gibert \& Pastor, 2007). Face à ces élèves en difficulté de lecture, la mise en place d'actions de remédiation s'impose. Si les programmes visant à améliorer la compréhension de textes sont les premiers auxquels on pense, des entraînements de plus bas niveau visant à pallier les défaillances dans l'identification des mots écrits ont également leur place. En effet, d'après Fayol, Morais et Rieben (2007), entre 10 et $15 \%$ des élèves fréquentant la $6^{\mathrm{e}}$ soit ne maitrisent pas totalement les procédures de décodage grapho-phonologique, soit n'ont pas suffisamment automatisé les mécanismes pour un accès direct au lexique orthographique. Évidemment, ces difficultés n'apparaissent pas avec l'entrée dans le secondaire et elles concernent bien souvent les mêmes élèves qui étaient déjà en situation difficile face à l'apprentissage du langage écrit dès la fin de la première année de primaire. C'est pourquoi le ministère de l'Éducation nationale a décidé de mettre en place une évaluation afin de repérer les élèves qui, à la suite de cette première année, présentent d'importantes difficultés en lecture, en production d'écrits et en mathématiques.

Initiée expérimentalement en 2004 et généralisée par la suite, l'évaluation des élèves de dernière année du cycle 2 (CE1) doit favoriser, dans le cadre plus étendu du plan de prévention de l'illettrisme, la mise en place des aides nécessaires à apporter avant la fin du cycle des apprentissages fondamentaux. Ainsi, en prenant comme référence les performances observées lors des évaluations de 2006 à ce niveau de la scolarité, trois profils ont pu être établis : les élèves qui présentent des difficultés qualifiées de lourdes (25\% de la population concernée) ou légères (30\%), et ceux qui n'ont pas de difficultés (45\%). À l'observation de ces chiffres, il est difficile de croire qu'un quart des élèves est en très grande difficulté au début du CE1 (et principalement en ce qui concerne l'apprentissage de la lecture). Mais, si l'outil proposé et les critères de repérage des difficultés ne paraissent pas encore totalement adaptés à l'objectif visé (pour une critique, voir le rapport de l'IGEN rédigé par Claus \& Megard, 2007), il présente cependant l'avantage de faire apparaître de très grandes disparités entre les élèves. C'est également ce que confirme une récente étude portant sur la prévalence des troubles d'apprentissage et menée auprès de plus de 1000 élèves de CE1 (Billard et al., 2008). La recherche qui est présentée ici s'inscrit dans ce cadre très général de la prévention de l'illettrisme et de l'aide susceptible d'être apportée aux élèves en difficulté de lecture. Elle décrit et analyse les effets de séances de soutien en lecture centrées sur le décodage et proposées à de faibles lecteurs de CE1.

\section{ASPECTS THÉORIQUES}

\section{Décodage et compréhension : deux composantes essentielles de la lecture}

L'acte de lire est une activité mentale complexe qui fait appel à différents domaines, soit spécifiques à cette activité particulière comme le traitement de l'information écrite, soit plus généraux et touchant alors à l'attention, la mémorisation, les connaissances générales ou bien encore aux aptitudes intellectuelles ou à la motivation. Sans pour autant ignorer l'importance que peut revêtir chacun des domaines considérés, précisons que dans le cadre de cette expérimentation, nous nous en tiendrons principalement aux traitements spécifiques. En ce sens, nous avons fait le choix d'un modèle théorique qui distingue deux composantes essentielles dans le traitement du langage écrit (Gough \& Tunmer, 1986) : d'une part, l'identification des mots écrits (grâce aux mécanismes de décodage ou par reconnaissance directe), d'autre part les processus de compréhension (modèle mis par exemple en avant par l'ONL, 1998 et rappelé par Fayol \& Morais, 2004).

Ajoutons que les différentes composantes de ce modèle ne sont pas indépendantes les unes des autres mais en interaction, ce qui revient à dire que si, pour lire, les deux composantes sont indispensables, aucune n'est suffisante. II convient aussi de ne pas oublier que la mobilisation des opérations nécessaires à l'activité de lecture compréhension s'effectue sous la contrainte d'un système de traitement de l'information à capacité limitée. En conséquence, un haut degré d'automatisation de reconnaissance des mots écrits est souhaitable afin de permettre au lecteur de consacrer la plus grande partie de ses ressources attentionnelles aux processus d'intégration syntaxique et sémantique et à la construction d'un modèle mental cohérent (sous réserve que le lecteur possède évidemment les capacités cognitives et linguistiques indispensables à la compréhension du message lu).

Bien que qualifiée de slogan par certains (voir par exemple Cèbe \& Goigoux, 2007) et trop réductrice 
par d'autres (Chauveau, 2007), cette définition de la lecture permet de distinguer comme seule « habileté » spécifique à l'acte de lire l'identification des mots écrits, la compréhension faisant pour sa part appel à des " habiletés " linguistiques en grande partie similaires à celles mises en œuvre lors de la compréhension du langage oral. S'il n'est pas question pour nous de revenir ici sur ce modèle particulier, signalons toutefois que certains auteurs (par exemple, Pearson, 2000) en proposent une version plus étendue en ajoutant une troisième composante, représentée par les stratégies de compréhension pouvant intervenir plus particulièrement lors de l'activité de lecture. En effet, au-delà des différences de syntaxe, de vocabulaire, d'aménagement du récit entre langue orale et écrite, la permanence de la trace rend possible un travail de relecture, de régulation de la vitesse dans la prise d'information, l'aménagement de pauses, etc. Néanmoins, une distinction aussi nette entre ce qui relève des activités de décodage et ce qui relève des activités de compréhension (pour une discussion à ce sujet, voir Rieben, 2006) va permettre de concevoir l'existence de différents profils de lecteurs (Aaron, 1991) :

- des élèves bons compreneurs et bon décodeurs. Ils ne posent en général aucun problème aux enseignants car leur apprentissage de l'écrit se déroule sans encombre ;

- des élèves éprouvant des difficultés spécifiques dans la reconnaissance des mots écrits mais avec un niveau satisfaisant en compréhension orale. II peut s'agir soit d'élèves dont les mécanismes d'identification sont en cours d'acquisition (c'est le cas des apprentis lecteurs), soit d'élèves présentant un déficit d'automatisation de ces mêmes mécanismes, soit encore d'enfants dyslexiques (pour ces derniers, il est alors question de trouble d'apprentissage et non plus de simple retard) ;

- des élèves éprouvant des difficultés non spécifiques à la lecture. C'est le cas de ceux ayant des problèmes de compréhension à l'oral et à l'écrit mais sans difficulté dans la reconnaissance des mots écrits ;

- des élèves affichant de faibles performances en reconnaissance des mots écrits mais également en compréhension, orale et écrite. En général, les difficultés se retrouvent alors dans tous les domaines.

Soulignons enfin qu'il existe des élèves sans problèmes de décodage, ni de compréhension orale mais avec un faible niveau de compréhension écrite. II s'agit le plus souvent de sujets présentant des difficultés propres au traitement de l'écrit, notamment un manque de contrôle au cours de l'activité de lecture (Rémond, 2003). La troisième composante («Stratcomp») du modèle de Pearson (2000) permet de prendre en compte cette dernière catégorie d'élèves.

Distinguer précisément ces profils permet ensuite de poser un diagnostic quant à l'origine possible des difficultés et de concevoir par la suite des programmes de remédiation ou de soutien différenciés, en lien avec les difficultés constatées. À titre d'exemple, il serait peu judicieux de proposer un travail centré sur la compréhension écrite sans s'être au préalable assuré que l'élève est suffisamment autonome vis-àvis de la reconnaissance des mots écrits. Cependant, ceci ne signifie pas que tout travail portant sur la compréhension est impossible : il convient simplement de le proposer à l'oral dans un premier temps, la compréhension passant de l'écoute de textes lus par l'enseignant ou d'autres élèves plus avancés à une compréhension en situation de lecture silencieuse plus autonome, dès lors que des capacités de décodage suffisantes le permettent (pour un exemple avec des prélecteurs, voir Bianco, Pellenq \& Coda, 2004).

\section{Quelques expériences d'entraînement}

Si la finalité de toute lecture est bien la compréhension, il est incontestable que l'identification des mots qui composent un texte écrit est, sans être suffisante, une condition nécessaire pour accéder à sa signification (Sprenger-Charolles \& Colé, 2005). Pour notre part, nous avons pu montrer que même en toute fin d'école primaire, il était possible d'améliorer significativement la compréhension en lecture des élèves en exerçant uniquement les procédures de reconnaissance des mots écrits (Chardon, 2000). Lors de cette expérimentation, suite aux passations de deux tests standardisés évaluant pour l'un le décodage, pour l'autre la compréhension écrite, un groupe d'une dizaine de faibles décodeurs, faibles compreneurs est constitué au sein de chacune des trois classes participant à l'étude. Deux de ces groupes bénéficient d'un programme intensif d'entraînement de neuf heures (décodage, phonologie, lecture orale), à raison de deux heures par semaine, le troisième groupe étant un groupe contrôle. À l'issue de l'entraînement, des progrès sont constatés en décodage mais également, et c'est le résultat majeur, en compréhension en lecture silencieuse.

Plus récemment, nous avons retrouvé des résultats assez proches en intervenant auprès d'apprentis 
lecteurs de cours préparatoire (CP), scolarisés en zone d'éducation prioritaire (Chardon, 2005). Trois classes participent à cette nouvelle expérience : l'une d'entre elles bénéficie d'un programme d'entraînement en lecture pendant qu'une autre reçoit un entraînement de type placebo (entraînement en mathématiques) et que la troisième sert de groupe contrôle. Le programme d'entraînement en lecture comprend treize séances de cinquante minutes, dispensées à raison d'une ou deux fois par semaine. Chaque séance comporte des exercices phonologiques, un travail sur la mise en correspondance des graphèmes et des phonèmes, des activités d'écriture et d'exploration haptique et visuo-haptique des lettres étudiées (1). Les résultats d'un premier post-test mettent en évidence une amélioration significative de la lecture de mots, du niveau de conscience phonologique et de la qualité de l'écriture pour le groupe entraîné. Toutefois, dans des épreuves de dictée et de compréhension, les trois classes ne se distinguent pas entre elles. Par contre, lors d'une évaluation différée effectuée deux mois après la fin de l'entraînement, le niveau de lecture de la classe entraînée, établie grâce à un test standardisé (Inizan, Inizan \& Bartout, 2002), est supérieur à celui des deux autres classes. Ainsi, comme lors de la recherche précédente, des interventions destinées à parfaire la rapidité et la précision de la reconnaissance des mots écrits participent à l'amélioration du niveau de lecture des élèves, même si les effets de ces interventions peuvent ne pas être immédiatement perceptibles.

En résumé, la maîtrise du décodage semble conditionner, au moins en partie, l'activité de lecture : au début de l'apprentissage, elle permet de lire les mots nouveaux et tend à rendre l'élève autonome par rapport au texte ; à terme, elle autorise une identification rapide et automatique de la plupart des mots écrits, rendant plus aisée la compréhension du texte lu. Pour favoriser le plus précocement possible cette maîtrise, un entraînement des " habiletés " métaphonologiques (et plus particulièrement métaphonémiques (2)) semble pertinent. Associé à la connaissance des lettres de l'alphabet et à l'enseignement des correspondances grapho-phonologiques, l'entraînement est encore plus efficace (Ehri et al., 2001, pour une revue). Ces entraînements peuvent prendre place très précocement dans le cursus scolaire de l'élève (dès la maternelle, avant l'apprentissage formel de la lecture qu'ils préparent et facilitent) mais également plus tard, en direction des faibles lecteurs dont ils améliorent le niveau de lecture, point qui nous intéresse particulièrement dans la recherche présentée ici.

\section{Sous quelle forme dispenser les entraînements ?}

Nous nous sommes cependant demandé de quelle façon dispenser des entraînements visant à exercer le décodage. Nous avons alors songé à la mise en œuvre possible de deux dispositifs, contrastés selon le caractère plus ou moins directif de la pédagogie mise en œuvre (donc la place accordée à l'enseignant pendant les séances) et le type d'organisation de la classe (élèves travaillant en groupe ou en individuel).

Le premier dispositif que nous avons déjà expérimenté lors de nos précédentes études peut être rattaché au domaine de l'enseignement direct ou explicite. Davantage fondée sur des résultats de recherches empiriques qu'issue d'une théorie de l'apprentissage particulière, cette approche fournit des conduites générales d'enseignement à adopter face aux élèves. Ainsi, lors d'une phase de présentation collective (en classe entière ou en grand groupe d'élèves), le maître fixe les objectifs de la leçon. II explicite ce qui est attendu et expose les notions qui seront travaillées lors de la séance. Dans une seconde phase, des exercices reprenant les notions étudiées sont proposés aux élèves qui peuvent travailler soit à nouveau en grand groupe, soit par équipe. Le maître dirige et contrôle le bon déroulement de ces exercices, s'assure de la compréhension de la tâche et apporte des informations supplémentaires si besoin. Lors d'une troisième phase, les élèves sont invités à travailler individuellement, en autonomie. Des révisions périodiques sont ensuite proposées pour stabiliser les apprentissages effectués. Cette démarche se révèle particulièrement efficace auprès des élèves en difficulté (Bissonnette, Richard \& Gauthier, 2005). Souvent confondue avec une démarche strictement transmissive, elle s'en écarte pourtant par l'importance accordée à la vérification de la compréhension des notions abordées en cours et non pas seulement en fin de séance, comme simple modalité de contrôle. À titre d'exemple et dans le domaine de la lecture, les résultats des travaux rapportés, d'une part par Torgesen (2002) pour la composante "identification des mots écrits ", d'autre part par Bianco et Bressoux (2009) pour la composante " compréhension ", montrent une réduction des difficultés à la suite d'interventions conduites avec une telle démarche. Nos propres résultats antérieurs vont également dans ce sens.

Le deuxième dispositif met l'accent sur les interactions entre les élèves et peut être rattaché au domaine de l'apprentissage coopératif. Après une 
première phase semblable à l'approche précédente (présentation des objectifs de la séance et des notions abordées), les élèves, rassemblés par petits groupes, travaillent de manière autonome. Ils sont invités à s'aider mutuellement, à justifier et à négocier les réponses qui leur sont demandées dans les différents exercices. De très nombreux travaux ont montré les avantages et les limites de telles organisations du travail de l'élève (pour des revues en français, voir par exemple Baudrit, 2005 ; Buchs, Lehraus \& Butera, 2006 ; Darnon, Butera \& Mugny, 2008). En ce qui concerne la lecture, la plupart des recherches faisant appel à l'apprentissage coopératif ont pour thème la compréhension et, en général, les résultats présentés sont positifs. Nous nous limiterons à évoquer ici l'une des plus connues en ce domaine, celle de Palincsar et Brown (1984). Dans le but de développer la compréhension en lecture, ces auteurs ont proposé des situations d'enseignement réciproque au sein de petits groupes d'élèves. À tour de rôle, chaque élève joue le rôle de l'enseignant en prenant en charge la lecture d'un paragraphe et en questionnant ses camarades. Ce faisant, il tente de mettre en œuvre différentes stratégies (identifiées comme efficaces chez les bons compreneurs) telles que poser des questions sur le texte lu, en clarifier les passages difficiles, prédire sa suite ou encore le résumer. Les autres élèves du groupe peuvent également poser des questions en vue d'obtenir ou d'apporter des compléments d'informations. Au début de la séquence, le guidage de l'enseignant est important, mais il doit normalement s'estomper au fil des séances. Les résultats montrent que cette méthode permet une amélioration de la compréhension de textes avec des élèves en difficulté d'apprentissage.

Plus récemment, et davantage en lien avec nos propres préoccupations centrées sur l'amélioration des mécanismes d'identification des mots, Lavoie, Levesque et Laroui (2008) ont analysé les interactions dans des situations d'écriture de 30 dyades d'élèves scolarisés en première année de primaire, en tenant compte de la constitution des paires (sexe et niveau de compétence en lecture) et du moment de l'année où les observations sont effectuées (octobre, février et mai). Leurs résultats indiquent qu'il n'y a pas de relation entre la façon dont les paires sont formées (genre, niveau à l'écrit) et la manière dont les élèves interagissent. Ils montrent également que les interactions sont davantage élaborées en début qu'en fin d'année. Ces résultats vont quelque peu à l'encontre de ce qui pouvait être attendu : généralement, le niveau des élèves en présence influe sur la qualité des interactions (les élèves faibles sont souvent ceux qui bénéficient le moins des relations d'aide) et le début d'année semble un moment moins favorable que la fin. À titre d'explication, les auteurs suggèrent que les compétences en écriture, plus faibles en début d'année, inciteraient les élèves à se soutenir davantage. Nous pouvons toutefois regretter que cette étude ne fournisse aucune indication au sujet de l'influence d'un tel dispositif sur le niveau de performance des élèves.

Auparavant, lors d'une recherche incluant deux classes de CP et deux classes de CE2, Fijalkow (1995) s'était déjà intéressé aux interactions entre jeunes élèves en analysant leurs comportements lors d'ateliers de lecture écriture. Les élèves ont été observés à plusieurs reprises, pendant les mois de mars et d'avril, en situation réelle de classe. Ces observations montrent que globalement les élèves consacrent la majeure partie de leur temps à des activités centrées sur la tâche et que, lorsqu'ils ont besoin d'une aide, ils s'adressent de préférence à leurs camarades avant de se tourner vers des aides extérieures. Elles indiquent également que plus le niveau de lecture est élevé, plus l'élève fait preuve d'autonomie, en limitant son recours aux aides extérieures. Si cette étude suggère qu'il est possible de faire travailler ensemble des élèves en cours d'apprentissage de la langue écrite, comme la précédente, elle ne dit par contre rien des avantages qu'il pourrait y avoir à utiliser ce dispositif, notamment en termes d'efficacité.

\section{Choix des dispositifs et hypothèses}

Devant le manque, à notre connaissance, de recherches empiriques ayant tenté d'améliorer les processus d'identification des mots écrits en mettant les élèves en situation de travail de groupe, nous avons pensé qu'il serait intéressant de tester l'efficacité de ce dispositif, comparativement à un dispositif plus classique au sein duquel l'élève travaille seul. Nous avons alors imaginé, pour un même entraînement, deux dispositifs très contrastés au niveau de l'organisation du travail :

- le premier dispositif sera qualifié d'« individuel " (IND). II consistera à dispenser l'entraînement sous la forme pédagogique la plus communément employée dans les classes dans lesquelles nous sommes intervenu : le maître garde le contrôle de la situation et du temps, propose les exercices, guide l'élève, etc. Nous nous distinguons toutefois des situations d'enseignement direct telles que nous les avons décrites supra en ne proposant pas de 
phases de travail en groupe, et ce pour conserver un fort contraste avec le deuxième dispositif ;

- le deuxième dispositif, au sein duquel les élèves seront regroupés par équipe de deux, sera qualifié de « dyadique » (DYA).

Pour tester d'une part l'efficacité de notre entraînement, d'autre part le dispositif pédagogique le plus pertinent pour le dispenser, nous avons choisi d'intervenir auprès de jeunes lecteurs de CE1 en faisant les hypothèses suivantes:

- des activités visant à perfectionner les mécanismes de décodage et de reconnaissance des mots écrits devraient améliorer le niveau de lecture (décodage et compréhension) ;

- conformément aux observations généralement faites lors d'entraînements à la compréhension, les performances des élèves travaillant par deux devraient être supérieures à celles des élèves travaillant seuls.

Soulignons ici que notre expérimentation n'a d'autre ambition que celle de fournir une contribution à l'élaboration et la mise en œuvre d'exercices de soutien au sein des classes. En ce sens, elle se veut éloignée des «querelles de méthodes » reposant sur une catégorisation manichéenne des pratiques enseignantes, de toute façon difficile à manier sur le plan de la recherche et ne reflétant que partiellement les pratiques réelles, les enseignants ne pouvant en aucun cas être considérés comme simples exécutants de méthode ou modèle pédagogique venus de l'extérieur de la classe (Paquay, 2008). Ainsi si les deux dispositifs proposés, par certains aspects, se rapprochent pour le premier des principes de l'enseignement direct et pour le second de ceux de l'apprentissage coopératif, ils ne peuvent et ne doivent être assimilés à ces principes.

\section{DESCRIPTION DE L'EXPÉRIENCE}

\section{Sujets}

Soixante-quatorze élèves répartis dans trois classes de CE1 ont participé à l'expérience (3). Ils sont scolarisés dans deux écoles primaires d'une même ville. Tous les milieux socioéconomiques sont représentés, avec toutefois une prédominance des milieux moyens, voire faibles.

\section{Épreuves des prétests et post-tests (évaluation à court terme)}

Le prétest s'est déroulé juste avant les vacances de Toussaint, à la fin du mois d'octobre, et le posttest a eu lieu en février, quelques jours avant les vacances d'hiver. Ces deux tests sont composés des cinq mêmes épreuves (à passation collective, excepté le test de l'Alouette). Ils permettent de recueillir et de comparer, avant et après la période d'entraînement, les performances des élèves en lecture orale, en orthographe et en compréhension en lecture silencieuse. À chaque fois, tous les élèves des trois classes ont été évalués. Préalablement à la présentation des épreuves, nous tenons à faire la remarque suivante : il est toujours difficile d'évaluer la compréhension écrite avec de jeunes élèves. Les épreuves disponibles diffèrent souvent dans leur forme (présence ou non d'un support imagé, court texte narratif ou phrases isolées, questions ouvertes ou fermées, réponses à donner par écrit ou cases à cocher, etc.). Aussi, afin d'appréhender au mieux cette composante, nous avons proposé trois tests standardisés («E 20 », « Lecsil », « Batcomp ») différents par leur présentation et le mode privilégié d'interrogation choisi et dont nous avons sommé les résultats (par la suite, nous désignerons cette épreuve composite sous l'appellation générique «Comp »).

\section{Compétence en lecture (E 20)}

Cette épreuve (Khomsi, 1990) est composée de vingt planches de quatre images, chaque planche proposant un énoncé constitué soit de phrases simples ou complexes, soit d'un microrécit. Elle permet d'estimer un niveau global de compréhension et est étalonnée pour être administrée à des élèves de la fin de grande section (en modalité orale) à la $4^{\mathrm{e}}$ de collège. L'allure est libre. Le temps accordé est au plus de trente minutes.

\section{Test de lecture silencieuse (Lecsil)}

Proposée par Aubret et Blanchard (1991), cette épreuve se compose de trois feuillets comportant chacun deux ou trois textes narratifs accompagnés de cinq ou six questions. Bien qu'initialement non prévue pour ce niveau (car étalonnée pour être présentée à des élèves du CE2 à la $3^{e}$ de collège), nous avons tout de même soumis cette épreuve à nos sujets parce qu'elle est très proche des situations d'évaluation de la compréhension qui sont généralement proposées aux élèves par les enseignants. Nous n'avons présenté que les deux premiers feuillets et 
réduit le temps de passation, proportionnellement à cette réduction (trente-trois minutes).

\section{Batelem-R : lecture d'un texte (Batcomp)}

Ce test fait partie d'une batterie d'épreuves pour l'école élémentaire (Savigny et al., 2001). II est destiné à apprécier le niveau de compréhension en lecture silencieuse. II se compose d'un texte narratif (que les élèves gardent à disposition pendant toute la durée de la passation) suivi de questions. L'élève y répond soit en repérant les bonnes réponses parmi des distracteurs, soit en complétant un passage dans lequel des mots ont été retirés. Ce travail s'effectue en temps libre.

\section{Batelem-R : évaluation orthographique (Ortho)}

Appartenant à la même batterie d'épreuves que la précédente (Batcomp), cette épreuve classique de dictée distingue trois aspects : la phonétique, l'usage et le respect des règles grammaticales, tous trois pris en compte dans le calcul du score final. Sont dictés par ordre croissant de difficulté des phonèmes, des syllabes puis de courtes phrases. Dans le cadre de cet article, seul le score global sera examiné.

\section{Alouette-R (Alou)}

Cette épreuve (voir Lefavrais, 2005) donne des indications sur le nombre de mots lus (indice $M$ ) et correctement lus (indice $\mathrm{C}$ ) par un élève placé pendant trois minutes en situation de lecture orale d'un texte composé de 265 mots. Aucun contrôle de la compréhension n'est effectué car c'est avant tout l'évaluation de l'efficacité du décodage qui est visée ici. Elle fournit également des renseignements sur la vitesse (indice CTL) et la précision de lecture (indice CM), ce dernier indice s'obtenant en mettant en rapport l'indice $C$, le nombre de mots lus correctement, et l'indice $M$, le nombre total de mots lus, le résultat obtenu étant multiplié par 100. À noter que pour les premiers niveaux scolaires, les indices $C$ et CTL sont pratiquement confondus, bien peu d'élèves parvenant à lire le texte en moins de trois minutes (aucun lors de cette expérience). Nous n'avons donc conservé que l'indice C, l'indice CTL étant davantage adapté aux élèves plus âgés.

Notre objectif étant ici de tester les effets d'un soutien de bas niveau sur la reconnaissance des mots écrits, et par contrecoup escompté sur la compréhension en lecture, il convient de s'assurer, afin de pouvoir observer des progrès à ce niveau, que nos sujets possèdent bien les capacités cognitives et linguistiques nécessaires. À cette fin, nous avons pro- posé deux épreuves supplémentaires (il serait en effet peu pertinent de n'exercer que les mécanismes de reconnaissance des mots écrits si les difficultés relèvent également d'un autre niveau). Elles n'ont été administrées qu'une seule fois, aux mêmes dates que le prétest.

\section{Compréhension orale (ECOSSE)}

L'objectif de cette épreuve est d'évaluer la compréhension d'énoncés (plus précisément de phrases) à l'audition (Lecocq, 1996). Nous en avons proposé une version restreinte, composée de vingt-huit items, choisis à partir des quatorze derniers blocs de l'épreuve (J à $\mathrm{W}$, deux items sélectionnés par bloc).

Niveau intellectuel (PMC, soit Progressive matrices couleur)

Les résultats de ce test de raisonnement logique qui permet une appréciation du niveau intellectuel et des capacités cognitives ont été pris en compte d'une part pour apparier les sujets des différents groupes, d'autre part pour repérer au sein de la population d'origine d'éventuels sujets en grande difficulté (Raven, Court \& Raven, 1998).

\section{Épreuves du post-test différé (évaluation à moyen terme)}

Afin de suivre l'évolution des performances des élèves, une partie des épreuves a été à nouveau administrée en toute fin d'année scolaire. Nous avons ainsi proposé le test de l'Alouette (Alou) et l'épreuve d'évaluation orthographique (Ortho). Pour tester la compréhension en lecture silencieuse, nous n'avons conservé que l'épreuve Lecsil (cette épreuve semblant d'une part avoir la meilleure finesse discriminative parmi les trois épreuves de compréhension que nous avions proposées lors des deux tests précédents, d'autre part être peu susceptible d'être concernée par un quelconque effet plafond). À noter que, comme pour les passations d'octobre et de février, l'ensemble des élèves est évalué à ce dernier test.

\section{Constitution et équivalence des groupes expérimentaux et du groupe contrôle}

\section{Les groupes expérimentaux IND et DYA}

Ces deux groupes sont issus de deux classes appartenant au même groupe scolaire. Dans chacune de ces classes, les quatorze plus faibles décodeurs ont d'abord été identifiés à l'aide de l'indice $\mathrm{C}$ du test de l'Alouette (indice correspondant au nombre de 
mots correctement lus en trois minutes) et ont été retenus pour constituer les deux groupes expérimentaux. Ensuite, afin de contrôler l'effet maître, chacun des groupes IND et DYA a été composé d'un nombre identique d'élèves (ici sept) provenant de chacune des deux classes. Cette contrainte, bien souvent ignorée dans de nombreuses recherches, est pour nous la condition indispensable pour tenter de contrôler l'effet maître. II semble en effet fondé de penser que le contexte didactique de la classe (représentations des enseignants quant à l'enseignement/ apprentissage de la lecture et choix didactiques qui peuvent en dériver, vitesse de présentation des notions...) peut avoir une influence non négligeable sur les performances des élèves (Bressoux, 1994). Nous avons également tenu compte du cas des élèves pressentis, à cette période de l'année, pour une prise en charge sur le temps scolaire par les enseignants spécialisés, membres du réseau d'aide : nous les avons équitablement répartis au sein des groupes. Enfin, et dans la mesure du possible, l'équilibre entre filles et garçons a été respecté (chacun des trois groupes comporte 6 filles et 8 garçons).

\section{Le groupe contrôle CONT}

Les quatorze élèves composant ce groupe ont été sélectionnés selon les mêmes critères que ceux qui ont présidé au choix des élèves des classes expérimentales. Ils appartiennent à une troisième classe, située dans un autre groupe scolaire de la même commune.

\section{Équivalence des groupes}

Nous avons systématiquement vérifié que les performances des trois groupes formés étaient équivalentes, et ce pour l'ensemble des épreuves proposées lors du prétest. À ce sujet, des tests de KruskallWallis visant à vérifier cette équivalence (mais également celle des performances des trois classes d'origine) ont été effectués. Toutes les valeurs $\mathrm{H}$ ainsi obtenues se sont révélées non significatives. Précisons toutefois que la vérification de l'équivalence des deux groupes expérimentaux et du groupe contrôle ne garantit pas, à coup sûr, l'absence d'un effet particulier de la didactique de la classe contrôle pendant la période s'écoulant entre le prétest et les deux posttests. Ce biais aurait pu être évité en prenant dans chacune des classes le même nombre d'élèves pour constituer chacun des groupes. Nous avons cependant renoncé à cette organisation pour au moins deux raisons : le risque d'avoir des groupes aux effectifs trop peu chargés pour pouvoir faire des observations valides, mais surtout la crainte de provoquer une trop grande désorganisation de l'emploi du temps des classes. Le montage expérimental choisi a ainsi garanti aux deux enseignants des classes expérimentales de disposer, à chacune des demi-journées consacrées à l'entraînement, d'une heure de cours avec la totalité des élèves.

Par ailleurs, nous avons vérifié à chaque test et pour chacune des épreuves que les élèves des trois classes qui ne participaient pas à l'expérimentation ne se distinguaient pas significativement entre eux (tests de Kruskall-Wallis). Ce dernier point va à l'encontre de l'existence d'un effet maître bien marqué et conforte a posteriori nos choix méthodologiques. Les caractéristiques des élèves composant les trois groupes apparaissent dans le tableau 1 (précisons que les élèves ayant obtenu de très faibles performances au test ECOSSE et/ou au test de raisonnement logique ont été écartés lors de la constitution des groupes).

\section{LES ENTRAÎNEMENTS DES DEUX GROUPES IND ET DYA}

Chaque entraînement est composé de onze séances à raison d'une séance d'une heure par semaine, à jour et heure fixes. Il a été dispensé dans une autre salle que les salles de classe habituelles. En tenant compte des contraintes des emplois du temps des enseignants, six séances ont pu être programmées de la rentrée des vacances de Toussaint au début des vacances de Noël, cinq de la reprise de janvier au début des congés d'hiver.

\section{L'entraînement du groupe IND}

Les élèves de ce groupe travaillent individuellement sous l'autorité de l'expérimentateur. La séance commence par plusieurs lectures d'un court texte écrit au tableau. Ces quelques lignes servent de support à la présentation des graphèmes et des phonèmes qui seront étudiés dans la suite de l'heure.

Dans un deuxième temps, une fiche de lecture appelée «Gammes 》 qui comporte soixante-quinze mots contenant «les sons et les lettres » du jour, numérotés et présentés en trois colonnes, est distribuée. Chaque élève est invité à lire oralement une partie des mots notés sur cette fiche. Après cette lecture, des exercices écrits et individuels sont proposés : outre une dictée de mots contenant les sons 
Tableau 1. - Caractéristiques et performances des élèves avant les entraînements, dans chacun des groupes

\begin{tabular}{|c|c|c|c|c|c|c|c|c|c|}
\hline & \multicolumn{3}{|c|}{ Groupe IND ( $\mathrm{n}=14)$} & \multicolumn{3}{|c|}{ Groupe DYA (n = 14) } & \multicolumn{3}{|c|}{ Groupe CONT $(n=14)$} \\
\hline $\begin{array}{l}\text { Âge moyen } \\
\text { des élèves }\end{array}$ & \multicolumn{3}{|c|}{7 ans et 6 mois } & \multicolumn{3}{|c|}{7 ans et 4 mois } & \multicolumn{3}{|c|}{7 ans et 5 mois } \\
\hline $\begin{array}{l}\text { Âge min, max, } \\
\text { médian }\end{array}$ & $\begin{array}{c}7 \text { ans } \\
\text { et } 1 \text { mois }\end{array}$ & $\begin{array}{c}7 \text { ans } \\
\text { et } 10 \text { mois }\end{array}$ & $\begin{array}{c}7 \text { ans } \\
\text { et } 7 \text { mois }\end{array}$ & $\begin{array}{c}6 \text { ans } \\
\text { et } 11 \text { mois }\end{array}$ & $\begin{array}{c}7 \text { ans } \\
\text { et } 10 \text { mois }\end{array}$ & $\begin{array}{c}7 \text { ans } \\
\text { et } 4 \text { mois }\end{array}$ & $\begin{array}{c}6 \text { ans } \\
\text { et } 10 \text { mois }\end{array}$ & $\begin{array}{c}8 \text { ans } \\
\text { et } 5 \text { mois }\end{array}$ & $\begin{array}{c}7 \text { ans } \\
\text { et } 5 \text { mois }\end{array}$ \\
\hline $\begin{array}{l}\text { Score moyen } \\
\text { (et écart type) } \\
\text { au test ECOSSE } \\
\text { (total : } 28 \text { ) }\end{array}$ & \multicolumn{3}{|c|}{$21,5(1,8)$} & \multicolumn{3}{|c|}{$22,4(1,6)$} & \multicolumn{3}{|c|}{$23,9(2,3)$} \\
\hline $\begin{array}{l}\text { Scores min, } \\
\text { max, médian }\end{array}$ & 19 & 25 & 21 & 20 & 26 & 22 & 19 & 26 & 24 \\
\hline $\begin{array}{l}\text { Score moyen } \\
\text { (et écart type) } \\
\text { au test PMC } \\
\text { (total : } 36 \text { ) }\end{array}$ & \multicolumn{3}{|c|}{$21,9(4,5)$} & \multicolumn{3}{|c|}{$22,8(5,1)$} & \multicolumn{3}{|c|}{$21,6(4,1)$} \\
\hline $\begin{array}{l}\text { Scores min, } \\
\text { max, médian }\end{array}$ & 14 & 26 & 23,5 & 16 & 29 & 22 & 17 & 29 & 21,5 \\
\hline $\begin{array}{l}\text { Indice C moyen } \\
\text { (et écart type) } \\
\text { sur un total } \\
\text { de } 265 \text { mots }\end{array}$ & \multicolumn{3}{|c|}{$61,6(17,0)$} & \multicolumn{3}{|c|}{$64,0(20,5)$} & \multicolumn{3}{|c|}{$63,1(29,7)$} \\
\hline $\begin{array}{l}\text { Indice C min, } \\
\text { max, médian }\end{array}$ & 25 & 82 & 65,5 & 29 & 105 & 61,5 & 21 & 106 & 68 \\
\hline
\end{tabular}

étudiés, il s'agit de recompositions de mots (à partir de lettres ou de syllabes), de choix entre différents graphèmes pour compléter des mots, etc. L'avancée du travail est contrôlée par l'expérimentateur qui apporte et commente les corrections dès la fin de chaque exercice. La séance se poursuit par un travail de repérage de mots correctement orthographiés : dans une liste de vingt-huit mots, chaque mot cible est présenté accompagné d'un distracteur comportant soit des inversions de lettres («question »/ «questoin "; " vinaigre »/« vianigre »), soit des substitutions de graphèmes par d'autres proches phonologiquement (« confiture »/«confidure»; «boutique »/ " poutique »). À partir de la sixième séance, un exercice supplémentaire est proposé : il consiste à se prononcer sur la similarité phonologique de deux mots présentés conjointement (« saison »/« saizon »; " chemin »/« chemain »). II y a vingt-huit paires de mots. Ces deux derniers exercices ne sont pas corrigés avec les élèves, mais les résultats obtenus leur sont communiqués d'une semaine sur l'autre. En toute fin de séance, et si le temps le permet, ont lieu des jeux de "chasse aux mots » avec le support de la fiche "Gammes ». L'expérimentateur énonce oralement un mot, les élèves le retrouvent le plus rapidement possible et donnent, soit oralement, soit par écrit sur une ardoise, son numéro dans les listes.

\section{L'entraînement du groupe DYA}

Au sein de ce dispositif, les élèves travaillent par paire. Sont associés deux élèves ayant obtenu sensiblement les mêmes performances à la tâche de lecture à voix haute au prétest. Cette égalité de performance entre les deux partenaires a été systématiquement recherchée afin de limiter l'émergence d'une relation d'autorité, l'élève le plus avancé pouvant parfois se comporter en expert. De ce seul point de vue, les dyades peuvent donc être considérées comme homogènes car nous n'avons par contre pas tenu compte, lors de leur constitution, des liens d'amitié, du sexe ou de la classe d'origine des élèves. 
Le matériel des séances (textes d'introduction, exercices, listes de lecture) et l'ordre de présentation des graphèmes et phonèmes sont strictement les mêmes que pour l'entraînement du groupe IND. Cependant, alors que chaque élève du groupe IND possède son propre matériel (une fiche, un crayon, une ardoise...), ce n'est pas le cas pour les élèves du groupe DYA où chaque équipe possède ce même matériel en exemplaire unique. De la sorte, nous pensons inciter aux échanges entre partenaires. À ce sujet, précisons que pendant les quatre premières séances, nous avons très fréquemment rappelé quelques règles à respecter lors d'un travail en équipe, n'hésitant pas pour cela à inviter les partenaires à des changements de rôle : qui écrit, qui commence à lire, qui tient d'abord le rôle du maître et pendant combien de temps ? L'exercice de dictée nous a grandement aidé dans cette démarche ; en effet, il est facile de se répartir les rôles à ce moment-là, l'un dictant les mots et corrigeant par la suite les erreurs, l'autre écrivant (4).

Ces quelques rappels de règles ou de consignes ont semble-t-il été profitables puisque, dès la cinquième séance, nous avons estimé que les élèves avaient acquis des habitudes de travail adéquates (respect mutuel, débuts d'échanges, absence de conflits ouverts, écoute de l'autre...) et, de ce fait, nous avons pu nous montrer beaucoup moins directif en leur déléguant presque complètement l'organisation du travail à réaliser. Ainsi, chaque équipe recevait en début de séance une pochette contenant l'ensemble des fiches et, sur deux bandes de papier protégées du regard, le texte de la dictée à effectuer. Comme pour l'entraînement du groupe IND, les exercices de recomposition de mots, de mots à compléter, etc. sont corrigés collectivement dès leur achèvement. Seuls les résultats des tout derniers exercices sont communiqués en début de séance suivante, sous forme de score annoncé publiquement. Par contre, la correction de la dictée s'effectue sans que l'expérimentateur intervienne : les élèves possèdent le texte original et procèdent par eux-mêmes aux rectifications nécessaires. En toute fin de séance, s'il reste un peu de temps, les équipes réalisent une "chasse au mot " au cours de laquelle chaque élève, à tour de rôle, interroge son partenaire.

\section{La classe contrôle}

L'enseignant de la classe contrôle ne reçoit aucune indication particulière concernant la conduite de sa classe. Hors les temps de passation des tests collectifs ou individuels, les élèves poursuivent donc le programme habituel du CE1. Signalons que, comme pour les deux classes expérimentales, l'enseignant n'a pas connaissance de la nature des tests effectués et du contenu des séances. L'ensemble des résultats des élèves ne sont communiqués qu'à l'issue du post-test différé.

\section{RÉSULTATS}

\section{Résultats à court terme}

\section{Efficacité de l'entraînement}

Les scores moyens et médians obtenus par les élèves aux différentes épreuves avant et après les entraînements sont présentés dans le tableau 2 (sont également indiqués les indices de dispersion).

Toutes les différences observées entre prétest et post-test sont positives et significatives : tous les scores moyens des groupes progressent, et ce dans toutes les épreuves. Ce résultat n'est pas surprenant dans la mesure où une période de quatre mois s'est déroulée entre les deux passations et l'augmentation des performances peut être attribuée, au moins pour une part, à la poursuite de l'enseignement en classe. Reste cependant à savoir si ces progressions sont identiques pour les trois groupes. Pour répondre à cette question, nous avons calculé les différences de progrès mesurées par les écarts de performance entre post-test et prétest. Les comparaisons statistiques sont présentées dans le tableau 3.

Notons que les différences statistiques présentes dans ce tableau révèlent toutes des progressions à l'avantage de l'un ou l'autre groupe expérimental. La première hypothèse que nous formulions au départ est vérifiée : avec des élèves faibles décodeurs, quelle que soit la modalité de soutien mise en œuvre, un entraînement portant sur les mécanismes de reconnaissance des mots écrits améliore ces mécanismes, mais également les performances en orthographe et en compréhension (lignes 1 et 2 du tableau 3). Par contre, la stabilité de l'indice CM traduit une stagnation de la précision de lecture : si les élèves entraînés lisent davantage de mots dans un temps donné (augmentation de l'indice C), la proportion d'erreurs commises au cours de cette lecture reste sensiblement la même pour tous. 
Tableau 2. - Indice C, indice CM et scores (et écarts types) obtenus par les élèves aux différents tests dans chacun des trois groupes, avant et après les entraînements

\begin{tabular}{|c|c|c|c|c|c|c|c|c|c|c|c|c|c|c|c|c|c|c|}
\hline & \multicolumn{6}{|c|}{ Groupe IND $(n=14)$} & \multicolumn{6}{|c|}{ Groupe DYA $(n=14)$} & \multicolumn{6}{|c|}{ Groupe CONT $(n=14)$} \\
\hline & \multicolumn{3}{|c|}{ Prétest } & \multicolumn{3}{|c|}{ Post-test } & \multicolumn{3}{|c|}{ Prétest } & \multicolumn{3}{|c|}{ Post-test } & \multicolumn{3}{|c|}{ Prétest } & \multicolumn{3}{|c|}{ Post-test } \\
\hline $\begin{array}{l}\text { Indice C moyen } \\
\text { sur un total }\end{array}$ & \multicolumn{3}{|c|}{$61,6(17)$} & \multicolumn{3}{|c|}{$105,1^{\star *}(24,5)$} & \multicolumn{3}{|c|}{$64,1(20,5)$} & \multicolumn{3}{|c|}{$104,5^{\star \star}(28,8)$} & \multicolumn{3}{|c|}{$63,1(29,7)$} & \multicolumn{3}{|c|}{$87,4^{\star \star}(36,5)$} \\
\hline $\begin{array}{l}\text { Indice C min, } \\
\text { max, médian }\end{array}$ & 25 & 82 & 65,5 & 58 & 160 & 102 & 29 & 105 & 61,5 & 56 & 155 & 105,5 & 21 & 106 & 68 & 36 & 146 & 86 \\
\hline $\begin{array}{l}\text { Indice CM moyen } \\
(\max =100)\end{array}$ & \multicolumn{3}{|c|}{$76,4(9,4)$} & \multicolumn{3}{|c|}{$85,2^{\star \star}(7)$} & \multicolumn{3}{|c|}{$79,3(8,5)$} & \multicolumn{3}{|c|}{$86,3^{\star \star}(7,1)$} & \multicolumn{3}{|c|}{$75,4(10,1)$} & \multicolumn{3}{|c|}{$81,5^{\star \star}(7,5)$} \\
\hline $\begin{array}{l}\text { Indice CM min, } \\
\text { max, médian }\end{array}$ & 61 & 91,2 & 76,9 & 63,7 & 94,6 & 86,7 & 59,4 & 89,1 & 80,4 & 66,7 & 93 & 89,3 & 56,8 & 87,5 & 78,6 & 68,7 & 92,7 & 81,8 \\
\hline $\begin{array}{l}\text { Score moyen } \\
\text { au test Comp } \\
\text { (total : 20) }\end{array}$ & \multicolumn{3}{|c|}{$6(2,5)$} & \multicolumn{3}{|c|}{$9,1^{\star *}(2,7)$} & \multicolumn{3}{|c|}{$6(2,2)$} & \multicolumn{3}{|c|}{$10,2^{* *}(1,8)$} & \multicolumn{3}{|c|}{$7(2,6)$} & \multicolumn{3}{|c|}{$8,8^{* \star}(3,3)$} \\
\hline $\begin{array}{l}\text { Scores min, } \\
\text { max, médian }\end{array}$ & 3 & 11 & 5 & 4 & 14 & 9,5 & 2 & 10 & 6 & 7 & 13 & 10 & 3 & 12 & 7 & 4 & 15 & 8,5 \\
\hline $\begin{array}{l}\text { Score moyen } \\
\text { au test Ortho } \\
\text { (total : 68) }\end{array}$ & \multicolumn{3}{|c|}{$29,9(10,4)$} & \multicolumn{3}{|c|}{$42,5^{\star \star}(8,6)$} & \multicolumn{3}{|c|}{$30,8(9)$} & \multicolumn{3}{|c|}{$42,9^{\star \star}(7,2)$} & \multicolumn{3}{|c|}{$31,8(11,6)$} & \multicolumn{3}{|c|}{$35,7^{\star \star}(11,2)$} \\
\hline $\begin{array}{l}\text { Scores min, } \\
\text { max, médian }\end{array}$ & 10 & 45 & 30,5 & 26 & 54 & 44 & 15 & 43 & 31,5 & 30 & 52 & 45 & 15 & 51 & 31 & 21 & 55 & 35 \\
\hline
\end{tabular}

Légende : ** significatif à $p \leq 0,01$, d'après le test de Wilcoxon. Lecture : les élèves du groupe IND lisent, en moyenne, davantage de mots au post-test (105,1 mots) qu'au prétest (61,6 mots). Cette progression est significative à $p \leq 0,01$.

Tableau 3. - Comparaison par paire des progrès réalisés par les trois groupes entre prétest et post-test

\begin{tabular}{|c|c|c|c|c|c|c|c|c|}
\hline & \multicolumn{4}{|c|}{ Test Alou } & \multirow{2}{*}{\multicolumn{2}{|c|}{ Test Comp }} & \multirow{2}{*}{\multicolumn{2}{|c|}{ Test Ortho }} \\
\hline & \multicolumn{2}{|c|}{ Indice C } & \multicolumn{2}{|c|}{ Indice CM } & & & & \\
\hline & $z$ & $\mathrm{p}$ & $z$ & $p$ & $z$ & $p$ & $z$ & $p$ \\
\hline $\begin{array}{l}\text { Comparaison par paire } \\
\text { groupe IND / groupe CONT }\end{array}$ & $-2,94$ & $0,003^{\star *}$ & $-0,44$ & 0,66 & $-2,25$ & $0,02^{*}$ & $-3,43$ & $0,0006^{* *}$ \\
\hline $\begin{array}{l}\text { Comparaison par paire } \\
\text { groupe DYA / groupe CONT }\end{array}$ & $-2,69$ & $0,007^{\star *}$ & $-0,05$ & 0,96 & $-2,99$ & $0,002^{* *}$ & $-2,67$ & $0,007^{\star *}$ \\
\hline $\begin{array}{l}\text { Comparaison par paire } \\
\text { groupe IND / groupe DYA }\end{array}$ & $-0,58$ & 0,56 & $-0,55$ & 0,58 & $-0,67$ & 0,50 & $-0,05$ & 0,96 \\
\hline
\end{tabular}

Légende : * significatif à $p \leq 0,05 ;{ }^{* *}$ significatif à $p \leq 0,01$, d'après le test de Mann et Withney. Lecture : pour l'indice $C$ du test de l'Alouette, lorsque nous comparons les écarts de performance du groupe IND entre les deux tests avec ceux du groupe CONT, nous observons que la progression du groupe IND est statistiquement supérieure $(z=-2,94 ; p=0,003)$. 


\section{Efficacité d'un dispositif pédagogique particulier}

À l'issue de l'entraînement, les deux groupes expérimentaux IND et DYA ne se distinguent pas significativement l'un de l'autre et ce dans toutes les épreuves (ligne 3 du tableau 3). La deuxième hypothèse est invalidée. Ainsi, que les élèves soient mis en situation de travailler individuellement ou par groupe de deux ne change rien aux résultats. II n'apparaît donc pas qu'une des deux formes pédagogiques mises en œuvre soit plus pertinente que l'autre.

\section{Résultats à moyen terme}

L'impact de l'entraînement est-il toujours présent en fin d'année scolaire ? Autrement dit, les écarts de performance entre les groupes entraînés et le groupe contrôle constatés en février sont-ils toujours visibles en juin ? Pour répondre à cette question, le tableau 4 présente les résultats des dernières évaluations; le tableau 5 fournit les comparaisons statistiques des progrès réalisés par les trois groupes entre le prétest et le post-test différé. Les modes de lecture de ces deux tableaux sont respectivement identiques à ceux des tableaux 2 et 3 .

L'examen du tableau 4 nous permet de constater, sans réel étonnement, que les élèves des trois groupes, et ce pour la totalité des épreuves, améliorent leurs performances entre octobre et juin (toutes les différences observées entre les deux tests sont statistiquement significatives). Cependant, les résultats obtenus par le groupe contrôle en fin d'année sont toujours inférieurs à ceux des deux groupes expérimentaux. À titre d'exemple, si les élèves composant chacun des trois groupes lisent davantage de mots en fin d'année, ceux du groupe CONT n'en lisent en moyenne que 96,4 tandis que leurs camarades des groupes IND et DYA en lisent respectivement 121,6 et 117,5 . Ces écarts de performance sont-ils pour autant significatifs ? La réponse est apportée par l'observation des comparaisons par paire rapportées dans le tableau 5.

Tableau 4. - Indice C, indice CM et scores (et écarts types) obtenus par les élèves aux différents tests dans chacun des trois groupes, lors du prétest et du post-test différé

\begin{tabular}{|c|c|c|c|c|c|c|c|c|c|c|c|c|c|c|c|c|c|c|}
\hline & \multicolumn{6}{|c|}{ Groupe IND $(n=14)$} & \multicolumn{6}{|c|}{ Groupe DYA $(n=14)$} & \multicolumn{6}{|c|}{ Groupe CONT $(n=14)$} \\
\hline & \multicolumn{3}{|c|}{ Prétest } & \multicolumn{3}{|c|}{ Post-test 2} & \multicolumn{3}{|c|}{ Prétest } & \multicolumn{3}{|c|}{ Post-test 2} & \multicolumn{3}{|c|}{ Prétest } & \multicolumn{3}{|c|}{ Post-test 2} \\
\hline $\begin{array}{l}\text { Indice C moyen } \\
\text { sur un total }\end{array}$ & \multicolumn{3}{|c|}{$61,6(17)$} & \multicolumn{3}{|c|}{$121,6^{\star \star}(30,2)$} & \multicolumn{3}{|c|}{$64,1(20,5)$} & \multicolumn{3}{|c|}{$117,5^{\star \star}(36,2)$} & \multicolumn{3}{|c|}{$63,1(29,7)$} & \multicolumn{3}{|c|}{$96,4^{\star \star}(39,8)$} \\
\hline $\begin{array}{l}\text { Indice C min, } \\
\text { max, médian }\end{array}$ & 25 & 82 & 65,5 & 69 & 167 & 126,5 & 29 & 105 & 61,5 & 60 & 175 & 118,5 & 21 & 106 & 68 & 41 & 175 & 94,5 \\
\hline $\begin{array}{l}\text { Indice CM moyen } \\
(\max =100)\end{array}$ & \multicolumn{3}{|c|}{$76,4(9,4)$} & \multicolumn{3}{|c|}{$86,5^{\star *}(6,8)$} & \multicolumn{3}{|c|}{$79,3(8,5)$} & \multicolumn{3}{|c|}{$88,6^{\star \star}(4,7)$} & \multicolumn{3}{|c|}{$75,4(10,1)$} & \multicolumn{3}{|c|}{$82,1^{\star *}(7,1)$} \\
\hline $\begin{array}{l}\text { Indice CM min, } \\
\text { max, médian }\end{array}$ & 61 & 91,2 & 76,9 & 67,6 & 95,2 & 86,5 & 59,4 & 89,1 & 80,4 & 81,1 & 97 & 89,5 & 56,8 & 87,5 & 78,6 & 70,2 & 90,7 & 80,1 \\
\hline $\begin{array}{l}\text { Score moyen } \\
\text { au test Lecsil } \\
\text { (total : 20) }\end{array}$ & \multicolumn{3}{|c|}{$1,2(1,6)$} & \multicolumn{3}{|c|}{$6,5^{\star \star}(3,5)$} & \multicolumn{3}{|c|}{$1,2(1,5)$} & \multicolumn{3}{|c|}{$7,5^{\star \star}(2,7)$} & \multicolumn{3}{|c|}{$1,3(1,7)$} & \multicolumn{3}{|c|}{$4,4^{\star \star}(3,2)$} \\
\hline $\begin{array}{l}\text { Scores min, } \\
\text { max, médian }\end{array}$ & 0 & 5,7 & 1 & 1 & 11,4 & 7,1 & 0 & 4,8 & 1 & 4,8 & 12,4 & 6,7 & 0 & 4,8 & 1 & 0 & 9,5 & 4,5 \\
\hline $\begin{array}{l}\text { Score moyen } \\
\text { au test Ortho } \\
\text { (total : 68) }\end{array}$ & \multicolumn{3}{|c|}{$29,9(10,4)$} & \multicolumn{3}{|c|}{$47,8^{\star \star}(8,6)$} & \multicolumn{3}{|c|}{$30,8(9)$} & \multicolumn{3}{|c|}{$49,3^{* \star}(9,4)$} & \multicolumn{3}{|c|}{$31,8(11,6)$} & \multicolumn{3}{|c|}{$40,3^{\star \star}(10,5)$} \\
\hline $\begin{array}{l}\text { Scores min, } \\
\text { max, médian }\end{array}$ & 10 & 45 & 30,5 & 29 & 60 & 47 & 15 & 43 & 31,5 & 31 & 68 & 52 & 15 & 51 & 31 & 27 & 57 & 40 \\
\hline
\end{tabular}

Légende : ** significatif à $p \leq 0,01$, d'après le test de Wilcoxon. 
Tableau 5. - Comparaison par paire des progrès réalisés par les trois groupes entre prétest et post-test différé

\begin{tabular}{|c|c|c|c|c|c|c|c|c|}
\hline & \multicolumn{4}{|c|}{ Test Alou } & \multirow{2}{*}{\multicolumn{2}{|c|}{ Test Lecsil }} & \multirow{2}{*}{\multicolumn{2}{|c|}{ Test Ortho }} \\
\hline & \multicolumn{2}{|c|}{ Indice C } & \multicolumn{2}{|c|}{ Indice CM } & & & & \\
\hline & z & $\mathrm{p}$ & $z$ & $p$ & z & $\mathrm{p}$ & z & $\mathrm{p}$ \\
\hline $\begin{array}{l}\text { Comparaison par paire } \\
\text { groupe IND / groupe CONT }\end{array}$ & $-2,2$ & $0,02^{*}$ & $-0,31$ & 0,75 & $-2,3$ & $0,02^{*}$ & $-3,08$ & $0,002^{* *}$ \\
\hline $\begin{array}{l}\text { Comparaison par paire } \\
\text { groupe DYA / groupe CONT }\end{array}$ & $-2,14$ & $0,03^{*}$ & $-1,35$ & 0,17 & $-2,95$ & $0,003^{\star *}$ & $-2,67$ & $0,007^{\star *}$ \\
\hline $\begin{array}{l}\text { Comparaison par paire } \\
\text { groupe IND / groupe DYA }\end{array}$ & $-0,44$ & 0,66 & $-0,29$ & 0,77 & $-0,64$ & 0,52 & $-0,34$ & 0,73 \\
\hline
\end{tabular}

Légende : * significatif à $p \leq 0,05$; ** significatif à $p \leq 0,01$, d'après le test de Mann et Withney.

Précisons que, comme lors de l'examen du tableau 3, toutes les différences significatives observées ici marquent des progressions de performance supérieures pour les deux groupes expérimentaux. Les différences observées en février à l'issue de l'entraînement, et à l'avantage des groupes IND et DYA, sont toujours présentes au mois de juin. Elles concernent la lecture orale (indice C), l'orthographe et la compréhension en lecture silencieuse. Cet effet à moyen terme peut être constaté quelle que soit la condition dans laquelle l'entraînement a été dispensé (les deux groupes expérimentaux ne se distinguant pas entre eux). Comme nous l'avions précédemment constaté, l'ensemble des élèves améliorent leur précision de lecture (indice $\mathrm{CM}$ ) sans qu'il soit possible de distinguer particulièrement l'un des trois groupes.

\section{Réduction des écarts de performance au sein de chaque classe}

Nous venons d'établir, grâce à la comparaison avec un groupe contrôle, que les onze heures de soutien proposées se révèlent profitables pour les deux groupes d'élèves faibles décodeurs qui en ont bénéficié, et que les progrès effectués semblent se maintenir, au moins à moyen terme. II peut être cependant intéressant de savoir si, à l'issue des séances, les élèves entraînés ont réussi, au sein même de leur classe d'origine, à réduire l'écart qui les séparait des meilleurs décodeurs avant l'entraînement.
Puisque nous avons examiné l'ensemble des élèves des deux classes expérimentales à chaque évaluation, nous sommes en mesure de répondre à cette interrogation en calculant les écarts moyens de performance entre les groupes de faibles décodeurs et les groupes d'élèves sans difficulté de décodage n'ayant pas été inclus dans le soutien. Ces calculs ont été effectués en regroupant, au sein de chaque classe, les scores des sept élèves de la condition IND et des sept élèves de la condition DYA (ce regroupement nous paraît légitime dans la mesure où nous avons montré que, quelle que soit la condition IND ou DYA, les effets de l'entraînement sont identiques). Les résultats apparaissent dans le tableau 6.

Commençons par préciser qu'une réduction des écarts peut être liée soit à un fort accroissement des performances des élèves les plus faibles, soit à un accroissement moindre ou nul (voire même une diminution) de celles des élèves les meilleurs. À notre avis, les tests proposés ici fournissent d'importantes marges de progression à tous les élèves et les résultats présentés peuvent donc être examinés, un risque d'effet plafond pouvant être écarté. Nous constatons ainsi, à peu de chose près, les mêmes évolutions dans les deux classes expérimentales et, mis à part l'indice $C$ qui a tendance à s'accroître, les écarts de performance entre bons et faibles décodeurs ont tendance à diminuer à l'issue de l'entraînement. Cependant, cette réduction n'étant significative que pour l'épreuve d'orthographe, l'entraînement ne semble s'avérer que partiellement capable de réduire les écarts initiaux entre élèves. 
Tableau 6. - Évolution des écarts moyens de performance entre les élèves ayant bénéficié de l'entraînement et ceux n'en ayant pas bénéficié

\begin{tabular}{|c|c|c|c|c|c|c|c|c|}
\hline & \multicolumn{4}{|c|}{ Classe expérimentale 1} & \multicolumn{4}{|c|}{ Classe expérimentale 2} \\
\hline & \multicolumn{2}{|c|}{ Prétest } & \multicolumn{2}{|c|}{ Post-test 2} & \multicolumn{2}{|c|}{ Prétest } & \multicolumn{2}{|c|}{ Post-test 2} \\
\hline & Entr. & Pas entr. & Entr. & Pas entr. & Entr. & Pas entr. & Entr. & Pas entr. \\
\hline $\begin{array}{l}\text { Indice C moyen } \\
\text { (sur } 265 \text { mots) }\end{array}$ & 55,5 & 102,2 & 118,4 & 180,9 & 70,1 & 120,2 & 131,3 & 194 \\
\hline $\begin{array}{l}\text { Écart moyen } \\
\text { de performance }\end{array}$ & \multicolumn{2}{|c|}{46,7} & \multicolumn{2}{|c|}{62,5} & \multicolumn{2}{|c|}{50,1} & \multicolumn{2}{|c|}{62,7} \\
\hline Évolution des écarts & \multicolumn{4}{|c|}{$+15,8$} & \multicolumn{4}{|c|}{$+12,6$} \\
\hline $\begin{array}{l}\text { Indice CM moyen } \\
(\max =100)\end{array}$ & 75,5 & 88,1 & 87,1 & 95 & 81,7 & 89,2 & 89,4 & 93,5 \\
\hline $\begin{array}{l}\text { Écart moyen } \\
\text { de performance }\end{array}$ & \multicolumn{2}{|c|}{12,6} & \multicolumn{2}{|c|}{7,9} & \multicolumn{2}{|c|}{7,5} & \multicolumn{2}{|c|}{4,1} \\
\hline Évolution des écarts & \multicolumn{4}{|c|}{$-4,7$} & \multicolumn{4}{|c|}{$-3,4$} \\
\hline $\begin{array}{l}\text { Score moyen } \\
\text { au test Lecsil } \\
\text { (total : 20) }\end{array}$ & 1,1 & 6,7 & 6,6 & 11,5 & 1,5 & 7,1 & 7,4 & 10,7 \\
\hline $\begin{array}{l}\text { Écart moyen } \\
\text { de performance }\end{array}$ & \multicolumn{2}{|c|}{5,6} & \multicolumn{2}{|c|}{4,9} & \multicolumn{2}{|c|}{5,6} & \multicolumn{2}{|c|}{3,4} \\
\hline Évolution des écarts & \multicolumn{4}{|c|}{$-0,7$} & \multicolumn{4}{|c|}{$-2,2$} \\
\hline $\begin{array}{l}\text { Score moyen } \\
\text { au test Ortho } \\
\text { (total : 68) }\end{array}$ & 28,8 & 46,2 & 46,2 & 58,2 & 32 & 44,6 & 51,1 & 56,9 \\
\hline $\begin{array}{l}\text { Écart moyen } \\
\text { de performance }\end{array}$ & \multicolumn{2}{|c|}{17,4} & \multicolumn{2}{|c|}{12} & \multicolumn{2}{|c|}{12,6} & \multicolumn{2}{|c|}{5,8} \\
\hline Évolution des écarts & \multicolumn{4}{|c|}{$-5,4^{*}$} & \multicolumn{4}{|c|}{$-6,8^{*}$} \\
\hline
\end{tabular}

Légende : * significatif à $p \leq 0,05$, d'après le test de Mann et Withney. Lecture : pour la classe expérimentale 1 , au prétest, les élèves entraînés (groupes IND et DYA réunis) ont correctement lu en moyenne 55,5 mots au test de l'Alouette (indice C) pendant que les meilleurs décodeurs de cette classe en lisaient 102,2. L'écart entre bons et faibles décodeurs est donc de 46,7 mots. Au post-test différé, cet écart est de 62,5 mots. II s'est donc accru de 15,8 mots, sans pour autant que cette évolution soit statistiquement significative.

\section{DISCUSSION}

L'objectif de cette recherche était d'évaluer l'effet d'un entraînement visant à améliorer, chez de faibles décodeurs de fin de cycle 2 , les mécanismes d'identification des mots écrits mais également la compréhension en lecture. Pour ce faire, onze séances centrées uniquement sur les aspects spécifiques de la lecture (décodage) ont été conduites en faisant appel à deux dispositifs pédagogiques contrastés afin d'en tester les efficacités respectives : un premier dispositif que nous avons qualifié d'individuel (IND), au sein duquel l'enseignant a un rôle actif et prépondérant dans la structuration des activités ; un deuxième dispositif supposé favoriser le travail de groupe et la coopération (DYA). Ainsi pour un même contenu, les élèves du groupe IND travaillaient individuellement sous l'autorité de l'expérimentateur et ceux du groupe DYA étaient regroupés par paire et pouvaient organiser librement leur travail (avec toutefois le soutien possible de l'expérimentateur, au moins lors des premières séances).

Les performances des élèves ont été mesurées en octobre et en février, avant et après chaque type d'entraînement, en utilisant des tests de compréhension écrite, un test de niveau orthographique et un 
test de lecture orale. En juin, quatre mois après la fin de l'entraînement, un post-test différé a été administré afin de s'assurer que les éventuels progrès constatés à l'issue du programme se maintenaient par la suite.

À court terme, les résultats des deux groupes expérimentaux IND et DYA attestent conjointement de l'impact favorable des séances sur la lecture orale, l'orthographe et la compréhension en lecture silencieuse. Cette première série de résultats vient confirmer ceux d'autres travaux que nous avons conduits et qui ont déjà montré que des programmes centrés sur le développement des mécanismes de reconnaissance des mots écrits et leur automatisation améliorent significativement à la fois la capacité de lecture orale mais également, et c'est le plus important, la compréhension en lecture (Chardon, 2000, 2005 ; Chardon \& Baillé, 2002). Reprenons maintenant plus en détail chacun des résultats obtenus.

À l'aide du test de l'Alouette, nous avons observé que si les élèves des groupes expérimentaux lisent davantage de mots que ceux du groupe contrôle (ils lisent donc plus vite), les pourcentages d'erreurs qu'ils commettent sont comparables. Cette absence d'effet sur la précision ne doit cependant pas masquer leur progression dans leur capacité à lire : en effet, lire en moyenne une vingtaine de mots en plus (ce qui correspond à un peu plus d'une ligne du test) atteste chez ces élèves d'une amélioration des mécanismes de décodage supérieure à celle de leurs homologues du groupe contrôle. Quand aux erreurs commises, il semble que tous les lecteurs débutants en commettent de façon inévitable lors de la phase d'apprentissage, l'ensemble de nos sujets n'échappant pas à cette règle. L'étalonnage du test montre d'ailleurs que l'indice de précision CM reste assez stable au niveau du CP et du CE1, avant d'effectuer un saut quantitatif relativement important entre sept et huit ans, au niveau du CE2, classe au-delà de laquelle cet indice évolue beaucoup moins. Quoi qu'il en soit, l'augmentation de la vitesse et du décodage phonologique ouvrent de nouvelles perspectives aux élèves, notamment lorsqu'ils sont confrontés à une épreuve de compréhension écrite. Lisant plus vite, ils parcourent une plus grande partie du texte et augmentent leurs possibilités de répondre correctement aux questions. C'est ce que montrent, selon nous, les progrès réalisés à l'épreuve de compréhension. Ces derniers résultats peuvent également être expliqués dans le cadre d'un système de traitement de l'information à capacité limitée : si la part d'attention que l'apprenti lecteur consacre à la reconnaissance des mots est allégée (grâce à une meilleure automaticité des procédures de décodage), il disposera de davantage de ressources à consacrer aux traitements relevant de la compréhension.

Concernant l'épreuve d'évaluation du niveau orthographique, rappelons tout d'abord que nous y avons eu recours car la dictée est une tâche susceptible de mettre au jour la capacité de nos élèves à transformer une information sonore en trace écrite, et donc à utiliser correctement les règles de correspondance entre les phonèmes et les graphèmes que nous avons étudiées avec eux lors des séances. Bien évidemment, certains des mots que nous avons dictés ont pu être notés à partir de leurs représentations stockées en mémoire, en faisant appel à une procédure directe, en partie automatisée. Cependant, d'un point de vue développemental, la production orthographique commence par prendre appui sur l'analyse phonologique des mots énoncés, c'est-à-dire sur une procédure indirecte et contrôlée (Zesiger \& Partz, 1997 ; Martinet \& Valdois, 1999). Or, vu l'âge et le niveau en lecture de nos sujets, nous pensons que la plus grande partie des mots dictés a été écrite en utilisant la procédure indirecte. Dans ce cadre, l'observation d'un impact positif laisse penser que notre proposition de travail systématique, centré sur les aspects phonologiques, a certainement participé à rendre plus efficaces les procédures d'analyse phonologique et de transcription des mots. Ce résultat est d'autant plus intéressant que ces progrès peuvent en engendrer d'autres, notamment en facilitant le recours aux connaissances orthographiques lors des transcriptions, lorsqu'on sait que le décodage des mots en lecture participe à la mémorisation de leurs formes orthographiques (Sprenger-Charolles, Béchennec \& Lacert, 1998).

À moyen terme, les impacts positifs observés à l'issue de l'entraînement sur la lecture orale (nombre de mots correctement lus), la compréhension et l'orthographe sont maintenus. Un programme de onze heures d'entraînement parvient donc à améliorer durablement le niveau de lecture des élèves. Pour autant, ces progrès permettent-ils aux plus faibles décodeurs de se rapprocher des scores des meilleurs ? Concernant l'épreuve d'orthographe, la réponse est sans conteste affirmative : les écarts moyens de performance se sont significativement réduits. Concernant les autres épreuves, les évolutions sont variables et moins tranchées : sans jamais atteindre de seuil significatif, elles vont dans le sens d'une augmentation des écarts pour le nombre de 
mots correctement lus et d'une réduction pour la précision de lecture et la compréhension.

En résumé, le programme d'entraînement dispensé a permis à de faibles décodeurs de progresser significativement par rapport à un groupe contrôle équivalent, montrant ainsi son efficacité. Son effet sur la réduction des écarts entre bons et faibles décodeurs au sein des classes est moins marqué mais bien réel pour au moins une épreuve. Cette réduction (ou cette relative stabilité) des écarts est à souligner : elle peut participer à minimiser l'apparition du phénomène connu sous le nom d'« effet Mathieu ». Décrit par Stanovich (1986), ce phénomène, du fait d'une exposition différentielle à la langue écrite, réside en une amélioration de l'« habileté » de lecture des bons lecteurs tandis que les lecteurs en difficulté voient la leur décroître. Autrement dit, si une pratique efficace de la lecture tend à favoriser, au travers de contacts fréquents avec les textes, des effets positifs sur les plans cognitif, linguistique et motivationnel, dans le même temps des difficultés précoces peuvent engendrer une suite d'effets négatifs sur les mêmes plans. Le développement du plaisir de lire, et donc de la fréquentation de l'écrit, peuvent en retour s'en trouver affectés. Ainsi, comme le préconise Rieben (2006), c'est en remédiant le plus tôt possible aux difficultés de lecture qu'il est peut-être possible de rompre cette spirale de l'échec.

Disons à présent quelques mots des deux dispositifs pédagogiques utilisés pour la mise en œuvre de notre soutien. Nous avions fait l'hypothèse, conformément à ce qui est généralement observé pour la compréhension en lecture, que le fait de regrouper les élèves (ici par deux) pour travailler le code serait plus bénéfique sur le plan des apprentissages (notamment la reconnaissance des mots écrits) qu'une situation plus classique, dans laquelle l'élève travaillerait seul. Il n'en est rien, aucun des deux dispositifs ne se montre plus efficace que l'autre, des progrès équivalents étant relevés dans toutes les dimensions testées. Comment expliquer cette absence de différence ? À notre avis, plusieurs raisons peuvent être invoquées.

La principale nous paraît liée au contenu même des séances. Habituellement, les tâches servant de support au travail de groupe sont complexes. Elles sont censées faire appel à des raisonnements de haut niveau, employés lors de résolutions de problèmes, d'activités métacognitives liées à la compréhension, etc. L'aide d'autrui est souvent souhaitable, voire indispensable. Or travailler à la révision des correspondances entre graphèmes et phonèmes, s'en- traîner à la lecture de mots, émettre des avis sur leur prononciation ou leur orthographe sont des exercices dont l'élève peut éventuellement venir à bout sans l'aide d'un partenaire. II n'y a donc pas a priori de raison obligée pour qu'il interagisse avec d'autres. Rappelons aussi qu'acquérir (ou parfaire) les mécanismes de reconnaissance des mots écrits ne peut s'apparenter à une activité de résolution de problème grâce à laquelle l'élève découvrirait les règles de prononciation. Celles-ci ne se «négocient 》 pas, elles sont arbitraires, données une fois pour toutes. De plus, les réponses à apporter à nos différents exercices sont attendues (un mot à recomposer, à écrire, à retrouver parmi des distracteurs...). De ce fait, le travail est très contraint et l'élève, devant le peu de place accordée à l'initiative personnelle, ne peut pas mettre en œuvre des stratégies très variées. Enfin, il suffit qu'un des deux partenaires de la dyade trouve rapidement le mot recherché ou la forme orthographique correcte pour que l'interaction prenne fin : s'agissant avant tout d'exercices de révision, les solutions étaient presque toujours à portée des élèves, même pour les plus faibles d'entre eux. Cette absence de nécessité de faire appel au partenaire pour réaliser la tâche peut être mise en lien avec les observations rapportées par Lavoie et ses collègues (2008) qui montrent que les interactions au sein de dyades d'élèves dans une situation d'écriture tendent à être plus élaborées lorsque le niveau de compétence des partenaires n'est pas trop élevé (ils éprouvent alors davantage le besoin de se soutenir). Ainsi, il semble que la situation mise en œuvre (rapport entre nature des tâches à effectuer et organisation dyadique) n'ait pas été susceptible de profiter pleinement aux élèves.

L'âge est également un facteur à ne pas négliger. À ce sujet, Piaget (1971) indique qu'une véritable coopération entre élèves ne peut avoir lieu avant qu'ils ne soient capables de se décentrer et de coordonner des points de vue divergents. Dans cette optique, coopérer ne deviendrait possible que lorsque l'attitude égocentrique serait moins prégnante, ce que Piaget situe vers l'âge de 7 ans, précisément l'âge de nos sujets (voir également Doise \& Mugny, 1981). En conséquence, les élèves du groupe DYA auraient bien interagi, mais sans véritable entraide et sans prise en compte de la parole de l'autre, chacun s'attachant à ses propres réponses à apporter aux exercices proposés. Cependant cette question d'âge, sans être totalement remise en cause, doit être nuancée : de nombreux auteurs soutiennent en effet que dès la maternelle les enfants peuvent commencer à interagir et travailler ensemble (voir par exemple Berzin, 2005). 
Enfin la relative nouveauté du dispositif a pu déstabiliser les élèves du groupe DYA, peu familiers des situations de travail par deux ou en petits groupes. Or faire évoluer les habitudes de travail afin de créer un climat propice à l'entraide et la coopération demande certainement plus qu'une dizaine d'heures, même si nous avons insisté auprès des élèves, lors des premières séances, sur les comportements à privilégier pour un travail interactif efficace.

Pour conclure, n'oublions pas toutefois que si les progrès du groupe DYA ne sont pas supérieurs à ceux du groupe IND, ils ne sont pas pour autant inférieurs ! II ne serait donc pas légitime de lire au travers de ces résultats une quelconque supériorité d'un des deux dispositifs pédagogiques, tous deux conduisant à des résultats identiques. Nous y voyons au contraire, pour un enseignant désireux de mettre en place un tel soutien en lecture au sein de sa classe, un élargissement de l'éventail des possibles et une occasion offerte de varier les situations de travail avec ses élèves. Comme il n'existe vraisemblablement pas une «bonne façon " d'enseigner (Paquay, 2008 ; Carette, 2008), il n'existe certainement pas une "bonne façon » de prévenir les difficultés ou d'y remédier. Ainsi, présenter ce même programme sur ordinateur pourrait être une autre piste, pour peu bien évidemment que, dans ces conditions particulières, son efficacité soit démontrée.

Saint-Cyr Chardon saint-cyr.chardon@ujf-grenoble.fr IUFM de l'académie de Grenoble, université Joseph-Fourier-Grenoble 1 et LSE, université Pierre-Mendès-France-Grenoble 2

\section{NOTES}

(1) Le terme haptique renvoie au domaine de la perception tactile manuelle et de l'exploration active des objets. Dans l'expérience rapportée ici, nous avions distribué aux élèves des lettres cursives, découpées dans des feuilles de papier de verre et collées sur un carton. Après un temps d'exploration libre, les élèves devaient suivre les contours des lettres avec l'index en respectant les sens d'écriture, d'abord les yeux ouverts (exploration visuo-haptique) puis les yeux fermés (exploration haptique).

(2) Les « habiletés » métaphonologiques sont souvent regroupées sous le terme générique de conscience phonologique qui peut être définie comme la capacité d'un sujet à identifier et manipuler intentionnellement les unités sonores de la langue. Ces unités peuvent être la rime (on parle alors d'« habileté " métarimique), la syllabe (métasyllabique) ou bien encore le phonème (métaphonémique)

(3) Nous remercions très vivement les professeurs des écoles AnneSophie, Nathalie et Éric, ainsi que leurs élèves. Sans leur bon accueil et leur très grande disponibilité, cette recherche n'aurait pas été possible. Nous remercions également les deux directeurs d'école, Sandrine et André, pour leur accord.
(4) Avec ces jeunes élèves de CE1, peu habitués à travailler par équipe, un cadrage de l'expérimentateur s'est révélé nécessaire lors des toutes premières séances. Ainsi, au-delà des instructions données pour chaque exercice, nous avons guidé le travail des élèves par quelques consignes plus spécifiques aux situations de travail de groupe. Ces consignes ont été énoncées collectivement lors de la première séance ou rappelées par la suite au sein de chaque équipe si nécessaire. En voici quelques-unes : " II existe une règle d'or pour faire ce travail : vous avez le droit de discuter entre vous, mais vous devez toujours parler à voix basse pour ne pas déranger les autres » " "Vous devez profiter du fait que vous êtes à deux pour essayer de mieux réussir le travail demandé "; "Si vous n'êtes pas d'accord entre vous, vous ne vous fâchez pas, vous essayez calmement de trouver une solution ensemble "; " Vous n'avez qu'une fiche pour deux, vous devrez donc faire ce travail ensemble et n'écrire à chaque fois qu'une seule réponse ; vous écrirez à tour de rôle "; "Pour la dictée, je vais désigner dans chaque groupe celui qui tiendra le rôle du maître et celui qui tiendra le rôle de l'élève; ensuite, vous changerez de rôle "; "Pour les gammes, vous allez lire un mot chacun ; votre coéquipier pourra vous corriger s'il pense que vous n'avez pas lu correctement ; il pourra aussi vous aider si vous n'arrivez pas à lire ».

\section{BIBLIOGRAPHIE}

AARON P. (1991). "Can reading disabilities be diagnosed without using intelligence tests? " Journal of learning disabilities, vol. 24, $\mathrm{n}^{\circ} 3$, p. 178-186.

AUBRET J. \& BLANCHARD S. (1991). L'évaluation des compétences en lecture. Paris : EAP.

BAUDRIT A. (2005). L'apprentissage coopératif. Origines et évolutions d'une méthode pédagogique. Bruxelles : De Boeck.

BERZIN C. (2005). «Interactions entre pairs et apprentissages à l'école maternelle. Le cas du tutorat : intérêt et limites ». Spirale, $n^{\circ} 36$, p. 7-15.

BIANCO M. \& BRESSOUX P. (2009). « Effet-classe et effetmaître dans l'enseignement primaire : vers un ensei- gnement efficace de la compréhension ". In X. Dumay \& V. Dupriez (dir.), L'efficacité dans l'enseignement : promesses et zones d'ombre. Bruxelles : De Boeck, p. 35-54.

BIANCO M., PELLENQ C. \& CODA M. (2004). « Enseigner des stratégies pour comprendre en moyenne section de maternelle ". Le langage et l'homme, vol. 39, $\mathrm{n}^{\circ} 2$, p. 69-86.

BILLARD C., FLUSS J., DUCOT C. et al. (2008). «Étude des facteurs liés aux difficultés d'apprentissage de la lecture. À partir d'un échantillon de 1062 enfants de seconde année d'école élémentaire ". Archives de pédiatrie, vol. 15, $\mathrm{n}^{\circ} 6$, p. 1058-1067. 
BISSONNETTE S., RICHARD M. \& GAUTHIER C. (2005). "Interventions pédagogiques efficaces et réussite scolaire des élèves provenant de milieux défavorisés ". Revue française de pédagogie, $\mathrm{n}^{\circ} 150$, p. 87-141.

BRESSOUX P. (1994). «Les recherches sur les effets-écoles et les effets-maîtres ". Revue française de pédagogie, $\mathrm{n}^{\circ} 108$, p. 91-137.

BUCHS C., LEHRAUS K. \& BUTERA F. (2006). "Quelles interactions sociales au service de l'apprentissage en petits groupes ? ". In P. Dessus \& E. Gentaz (dir.), Apprentissage et enseignement. Sciences cognitives et éducation. Paris : Dunod, p. 183-199.

CARETTE V. (2008). « Les caractéristiques des enseignants efficaces en question ". Revue française de pédagogie, $\mathrm{n}^{\circ} 162$, p. 81-93.

CÈBE S. \& GOIGOUX R. (2007). « Concevoir un instrument didactique pour améliorer l'enseignement de la compréhension en lecture ». Repères, n³5, p. 185-208.

CHARDON S.-C. (2000). "Expérience de soutien en lecture auprès de faibles lecteurs de fin de cycle 3 ». Revue française de pédagogie, $\mathrm{n}^{\circ} 130$, p. 107-119.

CHARDON S.-C. (2005). «Évaluation d'un entraînement à la lecture au cours préparatoire sollicitant les modalités visuelle auditive et haptique ». Revue française de pédagogie, $\mathrm{n}^{\circ} 153$, p. 93-107.

CHARDON S.-C. \& BAILLÉ J. (2002). «Soutien en lecture : prise en compte des méthodes d'apprentissage ". Revue française de pédagogie, $\mathrm{n}^{\circ}$ 139, p. 81-95.

CHAUVEAU G. (2007). Le savoir-lire aujourd'hui. Les méthodes de lecture et l'apprentissage de l'Écrit. Paris : Retz.

CLAUS P. \& MEGARD M. (2007). Note sur le suivi de la mise en œuvre de l'évaluation des élèves à l'entrée de la première année du cours élémentaire (CE1). Rapport $n^{\circ}$ 2007-030 de l'Inspection générale de l'Éducation nationale. Disponible au format PDF sur Internet à l'adresse suivante : <http://lesrapports.ladocumentation francaise.fr/BRP/074000353/0000.pdf> (consulté le 19 mai 2009).

DARNON C., BUTERA F. \& MUGNY G. (2008). Des conflits pour apprendre. Grenoble : Presses universitaires de Grenoble.

DOISE W. \& MUGNY G. (1981). Le développement social de l'intelligence. Paris : InterÉditions.

EHRI L., NUNES S., WILLOWS D. et al. (2001). «Phonemic awareness instruction helps children learn to read: evidence from the national reading panel's meta-analysis ». Reading research quarterly, vol. 36, n³, p. 250-287.

FAYOL M. \& MORAIS J. (2004). « La lecture et son apprentissage ". In Observatoire national de la lecture, L'évolution de l'enseignement de la lecture en France, depuis dix ans. Paris : ministère de l'Éducation nationale, de l'Enseignement supérieur et de la Recherche, p. 13-60.

FAYOL M., MORAIS J. \& RIEBEN L. (2007). « Le développement cognitif et la lecture au collège ». In Observatoire national de la lecture, La lecture au début du collège. Paris : ministère de l'Éducation nationale, de l'Enseignement supérieur et de la Recherche, p. 89-105. Disponible au format PDF sur Internet à l'adresse suivante : <http://www.bienlire.education.fr/04-media/ documents/onl_lecture_college.pdf> (consulté le 19 mai 2009).
FIJALKOW E. (1995). "Apprendre à lire-écrire : un processus social». Psychologie et éducation, $\mathrm{n}^{\circ}$ 21, p. 51-67.

GIBERT F. \& PASTOR J.-M. (2007). «Compréhension à l'écrit et à l'oral des élèves en fin d'école primaire. Évaluation 2003 ». Éducation et formations, $n^{\circ} 185$. Disponible au format PDF sur Internet à l'adresse suivante : <http:// media.education.gouv.fr/file/81/7/5817.pdf> (consulté le 19 mai 2009).

GOUGH P. \& TUNMER W. (1986). « Decoding, reading and reading disability ". Remedial and special education, vol. $7, \mathrm{n}^{\circ} 1$, p. 6-10.

INIZAN A., INIZAN A. \& BARTOUT D. (2002). Échelle composite, évaluation du savoir lire au cours préparatoire, révision 2002. Paris : EAP.

KHOMSI A. (1990). Épreuve d'évaluation de la compétence en lecture : E 20. Paris : ECPA.

LAVOIE N., LEVESQUE J.-Y. \& LAROUI R. (2008). « Interagir pour s'aider à écrire en première année du primaire ". Psychologie et éducation, $\mathrm{n}^{\circ}$ 1, p. 59-76.

LEACH J., SCARBOROUGH H. \& RESCORLA L. (2003). "Late-emerging reading disabilities". Journal of educational psychology, vol. 95, $\mathrm{n}^{\circ} 2$, p. 211-224.

LECOCQ P. (1996). L'ECOSSE : une épreuve de compréhension syntaxico-sémantique. Villeneuve-d'Ascq : Presses universitaires du Septentrion.

LEFAVRAIS P. (2005). Alouette-R. Paris : ECPA.

MARTINET C. \& VALDOIS S. (1999). « L'apprentissage de l'orthographe et ses troubles dans la dyslexie développementale de surface ". L'année psychologique, $n^{\circ} 99$, p. 577-622.

OBSERVATOIRE NATIONAL DE LA LECTURE (1998). Apprendre à lire au cycle des apprentissages fondamentaux : GS, CP, CE1. Analyses, réflexions et propositions. Paris : Odile Jacob et CNDP.

PALINCSAR A. \& BROWN A. (1984). «Reciprocal teaching of comprehension-fostering and comprehensionmonitoring activities ». Cognition and instruction, vol. 1, $\mathrm{n}^{\circ} 2$, p. 117-175.

PAQUAY L. (2008). «Y a-t-il UNE bonne façon d'enseigner? Mise en question de la thèse de C. Gauthier ». Les dossiers des sciences de l'éducation, $\mathrm{n}^{\circ} 19$, p. 157-169.

PEARSON P. (2000). «Comprehension instruction: apprenticing young readers in the art and science of reading ». Communication présentée au CIERA, Ann Arbor, $\mathrm{MI}$.

PIAGET J. (1971). Où va l'éducation ? Paris : DenoëlGonthier.

RAVEN J.-C., COURT J. \& RAVEN J. (1998). Progressive matrices couleur. Paris : EAP.

RÉMOND M. (2003). « Enseigner la compréhension : les entraînements métacognitifs ». In D. Gaonac'h \& M. Fayol (dir.), Aider les élèves à comprendre du texte au multimédia. Paris: Hachette, p. 205-232.

RÉMOND M. (2007). « La lecture des élèves français au collège : un état des lieux ». In Observatoire national de la lecture, La lecture au début du collège. Paris : ministère de l'Éducation nationale, de l'Enseignement supérieur et de la Recherche, p. 17-27. Disponible au format PDF sur Internet à l'adresse suivante : <http://www.bienlire. education.fr/04-media/documents/onl_lecture_college. pdf> (consulté le 19 mai 2009). 
RIEBEN L. (2006). Le $21^{e}$ siècle verra-t-il (enfin) la disparition des polémiques stériles sur l'apprentissage de la lecture ? Actes du séminaire de l'AIDEP, Neuchâtel, p. 9-26. Disponible au format PDF sur Internet à l'adresse suivante: <http://www.irdp.ch/publicat/ textes/062.pdf> (consulté le 19 mai 2009).

SAVIGNY M., BARBIER C., COUPEY LE ROY R. et al. (2001). Batelem- $R$, batterie d'épreuves pour l'école élémentaire. Cycle II et première année du cycle III. Paris : EAP.

SPRENGER-CHAROLLES L. \& COLÉ P. (2005). Lecture et dyslexie: approche cognitive. Paris: Dunod.

SPRENGER-CHAROLLES L., BÉCHENNEC D. \& LACERT P. (1998). "Place et rôle de la médiation phonologique dans l'acquisition de la lecture/écriture ". Revue française de pédagogie, $\mathrm{n}^{\circ} 122$, p. 51-67.

STANOVICH K. (1986). "Matthew effects in reading: some consequences of individual differences in the acquisition of literacy ". Reading research quarterly, vol. 21, $\mathrm{n}^{\circ} 4$, p. 360-406.

TORGESEN J. (2002). "The prevention of reading difficulties ». Journal of school psychology, vol. 40, $\mathrm{n}^{\circ} 1$, p. 7-26.

ZESIGER P. \& PARTZ M.-P. de (1997). « Neuropsychologie de l'orthographe ». In L. Rieben, M. Fayol \& C. Perfetti (dir.), Des orthographes et leur acquisition. Lausanne : Delachaux \& Niestlé, p. 57-76. 